Fatima Zehra Kanani*, Adnan Haider Kazmi y Bushra Kaleem

\title{
La métrica Sigma del sistema Alinity ci: estudio sobre 39 magnitudes químicas y de inmunoensayo
}

https://doi.org/10.1515/almed-2021-0025

Recibido 11-01-2021; aceptado 22-02-2021;

publicado en línea 16-04-2021

\section{Resumen}

Objetivos: La métrica Sigma es una forma útil y económica de verificar la calidad de las pruebas en los laboratorios clínicos. Alinity ci es un analizador (Abbott Diagnostics) lanzado recientemente, cuyo rendimiento aún no ha sido suficientemente estudiado. Calculamos el valor Sigma de 39 magnitudes químicas y de inmunoensayo de dos sistemas Alinity ci.

Métodos: Las métricas Sigma se derivaron de los estudios de validación del método. El coeficiente de variación (CV) se estimó siguiendo la guía CLSP EP 15. Se emplearon tres métodos para comprobar desviaciones: material de evaluación de rendimiento, comparación de métodos alternativos y prueba de linealidad. Se emplearon límites de error total permitido similares o inferiores a los de los estudios de referencia.

Resultados: Todas las magnitudes químicas, excepto el nitrógeno ureico en sangre (BUN), mostraron un valor Sigma $>6$ en uno o más niveles y métodos. Ninguna de las magnitudes estudiadas obtuvo <3 Sigma. Entre los electrolitos, el sodio obtuvo <3 Sigma en dos niveles en el método de evaluación de rendimiento, aunque alcanzó $>4$ Sigma en los otros dos métodos. Los niveles Sigma obtenidos fueron similares a los de estudios anteriores.

Conclusiones: Los valores de Sigma fueron aceptables en todas las magnitudes químicas, de inmunoensayo y electrolitos analizados con Alinity ci. La métrica Sigma es una herramienta objetiva, económica y extendida de control interno de la calidad. Calculamos la métrica Sigma de

*Autor para correspondencia: Fatima Zehra Kanani, Sección de patología química, Departamento de Patología, The Indus Hospital, Plot C-76, Sector 31/5, Opposite، Crossing, Darussalam Society Sector 39 Korangi, Karachi, Karachi, Sindh, Pakistan,

E-mail: fatima.kanani@tih.org.pk

Adnan Haider Kazmi, Sección de patología química, Departamento de Patología, The Indus Hospital, Karachi, Pakistan

Bushra Kaleem, Indus Hospital Research Centre, The Indus Hospital, Karachi, Pakistan numerosas pruebas de alto rendimiento. Es necesario evaluar el rendimiento de estas pruebas a largo plazo.

Palabras clave: especificaciones de rendimiento; exactitud; precisión; Seis Sigma; validación.

\section{Introducción}

La métrica Sigma es una herramienta de evaluación de calidad ampliamente utilizada en la industria, cuyo uso en los laboratorios clínicos se está extendiendo [1]. Esta herramienta se basa en el concepto de los defectos por millón de oportunidades y su objetivo es minimizar los errores en los procesos [2]. En el laboratorio clínico, se suelen emplear estadísticas de control interno y externo de calidad para calcular valores mensurables para los cálculos Sigma, a partir de datos de exactitud y precisión de la prueba. Analistas clínicos y otros profesionales de laboratorio emplearon distintos métodos basados en esta técnica para evaluar y verificar el rendimiento de su laboratorio. La precisión se suele calcular mediante el coeficiente de variación generado a partir del análisis diario de los diferentes niveles de control interno de calidad empleados para las pruebas. La exactitud se estudia calculando la desviación, normalmente a partir de valores de grupos de comparación sobre esquemas de pruebas de rendimiento, aunque también se han utilizado otros métodos [3, 4]. Otra variable en el cálculo de la métrica Sigma es el límite de error total permitido (TE $\alpha)$. Se han propuesta diversos TE $\alpha$ para distintas magnitudes basados en el rendimiento analítico óptimo [5-8], las recomendaciones clínicas [9] o la variación biológica [10]. En la Tabla Suplementaria S1 se muestra un resumen de algunas de las fuentes actualmente disponibles.

La principal ventaja de la métrica Sigma es que se trata de una herramienta cuantificable y mensurable, que permite la evaluación y seguimiento objetivo del rendimiento de una prueba. Seis Sigma y superiores son la clase estándar mundial, en la que hay menos de cuatro defectos por millón de oportunidades. El nivel mínimo aceptable para una prueba analítica clínica es el nivel tres Sigma. Este nivel se aproxima a los 67.000 defectos por millón de 
oportunidades, por debajo del cual las pruebas no se consideran seguras clínicamente para realizar análisis de muestras de pacientes [11-13].

Otra ventaja importante de calcular la métrica Sigma de las pruebas analíticas es que permite medir objetivamente la calidad de las pruebas, pudiendo así espaciar la frecuencia de los controles internos de calidad. Una prueba con una alta precisión y exactitud, que arroja valores de Sigma elevados, requiere un menor número de controles internos de calidad por serie, más muestras por serie y menos reglas Westgard que aplicar. Por otro lado, las pruebas de mayor precisión o desviación requieren más controles internos de calidad, series más pequeñas, menos muestras y más reglas Westgard que aplicar. Este es el concepto por el que aboga Westgard en su última versión de Reglas de Westgard Sigma $[14,15]$. Esta herramienta reduce la carga de trabajo y los costes de las pruebas ordinarias, con un enfoque más centrado en las pruebas que suelen dar más problemas. El coste de las pruebas de laboratorio es una de sus principales limitaciones, no solo para las regiones menos desarrolladas, sino también para los laboratorios comerciales de todo el mundo. La crisis económica que está empezando a afectar a diversos países, especialmente ante la actual pandemia, ha evidenciado que las organizaciones necesitan desarrollar estrategias para reducir los costes de sus operaciones. La métrica Sigma es una de dichas estrategias.

En este estudio, empleamos tres métodos diferentes para calcular la métrica Sigma de la serie Alinity ci, en la que se han calculado las desviaciones a partir de datos de materiales de evaluación de rendimiento. Calculamos la métrica Sigma de las pruebas realizadas con el sistema Alinity ci durante las validaciones iniciales, lo cual forma parte de un ejercicio continúo orientado a adaptar el uso de controles internos de calidad.

\section{Materiales y métodos}

Dado que el presente estudio no estaba relacionado con la experimentación en animales o seres humanos, estaba exento de aprobación por parte del Comité Ético correspondiente. Se analizaron 39 parámetros en dos analizadores Alinity c y Alinity i entre los meses de junio y septiembre de 2018. Se conectaron dos analizadores Alinity ci a un sistema de automatización a3600, incluyendo los procesos pre y postanalíticos, como parte del sistema de automatización total del laboratorio químico clínico central. Los datos se generaron durante los ejercicios de validación del método, realizados con estos analizadores.

Abbott Diagnostics lanzó al mercado la plataforma analítica Alinity en 2017. Alinity c es un analizador químico clínico de electrolitos totalmente automatizado con electrodos selectivos de ión indirecto sobre tecnología de chip integrado (ICT, por sus siglas en inglés). Por otro lado, el sistema Alinity i es un analizador de inmunoensayo basado en micropartículas paramagnéticas y detección quimioluminiscente basada en un conjugado marcado con éster de acridinio. Se incluyeron los siguientes parámetros químicos clínicos y electrolitos: albúmina, fosfatasa alcalina (ALP), alanina aminotransferasa (ALT), amilasa, aspartato aminotransferasa (AST), nitrógeno ureico en sangre (BUN), calcio, cloruro, colesterol, $\mathrm{CO}_{2}$, creatinina fosfoquinasa (CPK), creatinina, bilirrubina directa, gamma-glutamil transferasa (GGT), glucosa, colesterol de lipoproteínas de alta densidad (C-HDL) hierro, lactato deshidrogenasa (LDH), colesterol de lipoproteínas de baja densidad (C-LDL), magnesio, fósforo, potasio, sodio, bilirrubina total, proteína total, triglicéridos y ácido úrico. Por otro lado, los parámetros de inmunoensayo incluidos fueron alfa-fetoproteína (AFP), gonadotropina coriónica humana beta ( $\beta$ -HCG), triyodotironina libre (FT 3), libre tiroxina (FT 4), ferritina, hormona estimulante del folículo (FSH), hormona luteinizante (LH), prolactina, hormona estimulante del tiroides (TSH), antígeno prostático específico total (PSA) y vitaminas B12 y D. Se calculó la métrica Sigma de todas las magnitudes en los dos conjuntos de analizadores y se consideraron los valores medios para el propósito del estudio. Las métricas Sigma se calcularon aplicando la siguiente fórmula:

$\boldsymbol{\Sigma} \boldsymbol{\sigma}=(\mathrm{TE} \alpha-$ desviación $) / \mathrm{CV}$ (donde $\Sigma \sigma$ es el valor de Sigma, TE $\alpha$ es el error total permitido y CV es el coeficiente de variación).

A. Error total permitido: Todos los TEas se extrajeron de la Ley de Mejora de Laboratorios Clínicos (CLIA), el Colegio de Patólogos Americanos (CAP) y el Programa Nacional de Educación sobre el Colesterol (NCEP), a excepción de la vitamina D, cuyo TE $\alpha$ se extrajo de un estudio de referencia sobre variaciones biológicas (Tabla S1) [10]. Se emplearon valores TE $\alpha$ similares o inferiores a los utilizados en estudios comparables [16, 17].

B. Precisión: Se aplicaron tres niveles de control de calidad en cinco réplicas durante cinco días en los dos sistemas Alinity ci, según las recomendaciones CLSI EP 15 [18]. Finalmente, se calculó el coeficiente de variación (CV\%).

C. Desviación: Se emplearon tres métodos diferentes para calcular la desviación:

\section{Evaluación de rendimiento}

Previamente, se analizó y almacenaron a $-40{ }^{\circ} \mathrm{C}$ los materiales de evaluación de rendimiento aplicando entre siete y diez niveles establecidos por el Colegio de Patólogos Americanos (CAP). Estos materiales se analizaron en los analizadores Alinity c e i en tres réplicas. Los resultados se compararon con la media del grupo comparable de Abbott Architect, ya que, en el momento del estudio, Alinity aún no había sido incluido en los estudios del CAP. La fórmula empleada para calcular la desviación fue [(media objetivo - media observada/media objetivo) ^100]. La desviación acumulada se obtuvo a partir de la media de todas las desviaciones.

\section{Experimento de correlación del método}

El experimento de correlación del método lo realizaron Roche Cobas c311[(Roche Diagnostics International Ltd, Rotkreuz, Suiza) para las magnitudes biológicas], Nova 16 [(Nova Biomedical Corporation 200 Prospect Street Waltham, Massachusetts, USA) para el sodio, potasio, cloruro, y $\mathrm{CO}_{2}$ total], Vitros Eci [(Ortho Clinical Diagnostics, Rochester, NY) para $\beta$-HCG, ferritina, FSH, FT3, FT4, LH, prolactina, TSH y PSA total] y Elecsys e411 [(Roche Diagnostics International Ltd, Rotkreuz, Suiza) para la vitamina D, vitamin B12, alfafetoproteína, hormona paratiroidea intacta y el sistema Alinity ci]. Se analizaron 40 muestras con el método existente, y se reanalizaron en las dos horas posteriores con los dos sistemas Alinity ci. 
Tabla 1: CV y desviación de magnitudes según dos métodos.

\begin{tabular}{|c|c|c|c|c|c|}
\hline \multirow[t]{2}{*}{ Magnitudes } & \multicolumn{3}{|c|}{$\mathrm{CV}, \%$} & \multicolumn{2}{|c|}{ Desviación, \% } \\
\hline & L1 & L2 & L3 & Método de evaluación de rendimiento & Comparación de métodos alternativos \\
\hline \multicolumn{6}{|c|}{ Analitos químicos clínicos } \\
\hline ALB & 1,062 & 0,835 & 0,599 & 3,49 & 1,41 \\
\hline ALP & 2,202 & 1,623 & 1,413 & 5,34 & 0,29 \\
\hline ALT & 1,687 & 1,776 & 0,966 & 2,78 & $-0,53$ \\
\hline AMI & 2,289 & 0,913 & 0,694 & 3,59 & $-1,07$ \\
\hline AST & 1,628 & 1,046 & 0,876 & 5,52 & $-0,64$ \\
\hline BUN & 1,808 & 1,923 & 1,548 & $-1,77$ & 1,16 \\
\hline $\mathrm{Ca}$ & 1,221 & 0,961 & 0,967 & $-0,75$ & $-0,27$ \\
\hline $\mathrm{COL}$ & 1,030 & 0,980 & 0,990 & $-0,47$ & $-0,46$ \\
\hline $\mathrm{CO}_{2}$ & 3,204 & 3,507 & 4,871 & 1,22 & 1,94 \\
\hline CPK & 1,273 & 0,740 & 0,691 & 14,01 & 1,86 \\
\hline CREA & 3,109 & 1,726 & 0,977 & 2,38 & 2,98 \\
\hline $\mathrm{BD}$ & 1,512 & 2,490 & 1,172 & $-0,40$ & 0,85 \\
\hline GGT & 1,886 & 0,908 & 1,002 & 9,57 & $-1,20$ \\
\hline GLU & 0,975 & 0,538 & 0,335 & 1,15 & $-0,34$ \\
\hline HDL-C & 1,675 & 0,791 & 0,962 & 4,21 & $-1,65$ \\
\hline Hierro & 1,052 & 0,627 & 0,910 & 3,18 & $-2,36$ \\
\hline LDH & 1,042 & 0,988 & 0,781 & 4,27 & $-0,50$ \\
\hline LDL-C & 0,950 & 0,643 & 1,435 & 1,31 & $-1,75$ \\
\hline Mg & 1,996 & 1,719 & 1,725 & $-2,85$ & $-1,21$ \\
\hline$P$ & 2,028 & 1,337 & 0,974 & $-0,69$ & $-0,22$ \\
\hline BT & 0,916 & 2,195 & 2,174 & 0,63 & $-0,17$ \\
\hline TG & 2,145 & 0,943 & 0,853 & $-3,13$ & 2,16 \\
\hline PT & 0,725 & 0,772 & 0,689 & 0,47 & $-0,03$ \\
\hline $\mathrm{AU}$ & 2,124 & 0,922 & 0,814 & $-0,43$ & $-0,42$ \\
\hline \multicolumn{6}{|c|}{ Inmunoensayo } \\
\hline AFP & 3,351 & 3,123 & 3,230 & $-0,26$ & $-1,62$ \\
\hline$\beta H C G$ & 4,382 & 4,158 & 2,198 & $-4,35$ & 0,77 \\
\hline FER & 2,839 & 2,725 & 2,606 & $-5,65$ & $-10,53$ \\
\hline FSH & 1,890 & 3,047 & 2,510 & $-0,05$ & $-3,49$ \\
\hline T3 libre & 2,744 & 3,420 & 3,164 & $-6,64$ & $-3,33$ \\
\hline T4 libre & 2,973 & 1,239 & 2,513 & $-5,83$ & 1,79 \\
\hline LH & 2,063 & 2,762 & 2,360 & 6,50 & $-4,63$ \\
\hline Prolactina & 2,241 & 2,667 & 2,667 & 3,64 & 3,11 \\
\hline PSA total & 1,370 & 1,910 & 2,274 & $-3,46$ & $-7,30$ \\
\hline TSH & 3,157 & 4,085 & 2,767 & 6,14 & $-2,22$ \\
\hline VIT B12 & 5,061 & 3,709 & 4,640 & $-2,93$ & $-0,07$ \\
\hline VIT D & 4,833 & 4,952 & 3,337 & $-3,90$ & $-6,40$ \\
\hline \multicolumn{6}{|l|}{ Electrolitos } \\
\hline $\mathrm{Cl}$ & 0,647 & 0,706 & 0,583 & 1,51 & 1,42 \\
\hline $\mathrm{K}$ & 1,856 & 1,173 & 0,842 & 0,43 & 0,41 \\
\hline $\mathrm{Na}$ & 0,766 & 0,537 & 0,737 & 1,58 & $-0,07$ \\
\hline
\end{tabular}

ALB, albúmina; ALP, fosfatasa alcalina; ALT, alanina aminotransferasa; AMI, amilasa; AST, aspartato aminotransferasa; Ca, calcio; COL, colesterol; $\mathrm{CO}_{2}$, dióxido de carbono; $\mathrm{CPK}$, creatinina fosfoquinasa; CREA, creatinina; $\mathrm{BD}$, bilirrubina directa; GGT, gamma glutamil transferasa; GLU, glucosa; HDL, lipoproteína de alta densidad; LDH, lactato deshidrogenasa; LDL, lipoproteína de baja densidad; Mg, magnesio; P, fósforo; PSA total, antígeno prostático específico total; BT, bilirrubina total; TG, triglicéridos; PT, proteína total; AU, ácido úrico; AFP, alfa fetoproteína; BHCG, gonadotropina coriónica humana beta; FER, ferritina; FSH, hormona estimulante del folículo; T3 libre, triyodotironina libre; T4 libre, tiroxina libre; HL, hormona luteinizante; TSH, hormona estimulante de la tiroides; VIT B12, vitamina B12; VIT D, vitamina D; Cl, cloruro; K, potasio; $\mathrm{Na}$, sodio.

\section{Experimento de linealidad}

Se realizaron estudios de linealidad en todas las pruebas en tres réplicas aplicando siete niveles y empleando material de linealidad comercial (magnitudes químicas y electrolitos) (o muestras de pacientes) (inmunoensayo), exceptuando las magnitudes con calibradores multipunto. La desviación se calculó a partir de la diferencia entre los valores esperados y la media de los valores observados, tal como se ha indicado anteriormente. Las desviaciones y valores de Sigma obtenidos en el experimento de linealidad se muestran en la Tabla Suplementaria S2. 
Tabla 2: Análisis de métrica de Sigma de magnitudes mediante varios métodos utilizando Alinity ci.

\begin{tabular}{|c|c|c|c|c|c|c|c|c|}
\hline \multirow[t]{2}{*}{ Magnitudes } & \multirow[t]{2}{*}{ TE $\alpha$} & \multirow[t]{2}{*}{ FUENTE } & \multicolumn{3}{|c|}{ Método de rendimiento } & \multicolumn{3}{|c|}{ Comparación de métodos alternativos } \\
\hline & & & L1 & $\mathbf{L} 2$ & L3 & L1 & L2 & L3 \\
\hline \multicolumn{9}{|c|}{ Química clínica } \\
\hline ALB & $10 \%$ & CLIA & 6,13 & 7,79 & 10,86 & 8,09 & 10,29 & 14,33 \\
\hline ALP & $30 \%$ & CLIA & 11,20 & 15,19 & 17,45 & 13,49 & 18,30 & 21,03 \\
\hline ALT & $20 \%$ & CLIA & 10,21 & 9,70 & 17,84 & 11,54 & 10,96 & 20,16 \\
\hline AMI & $30 \%$ & CLIA & 11,54 & 28,92 & 38,07 & 12,64 & 31,67 & 41,69 \\
\hline AST & $20 \%$ & CLIA & 8,89 & 13,84 & 16,52 & 11,89 & 18,50 & 22,10 \\
\hline BUN & $9 \%$ & CLIA & 4,00 & 3,76 & 4,67 & 4,34 & 4,08 & 5,06 \\
\hline $\mathrm{Ca}$ & $9,72 \%$ & CLIA & 7,34 & 9,33 & 9,28 & 7,74 & 9,83 & 9,78 \\
\hline $\mathrm{COL}$ & $9 \%$ & NCEP & 8,28 & 8,80 & 8,62 & 8,29 & 8,71 & 8,63 \\
\hline $\mathrm{CO}_{2}$ & $25 \%$ & CAP & 7,42 & 6,78 & 4,88 & 7,20 & 6,57 & 4,73 \\
\hline CPK & $30 \%$ & CLIA & 12,56 & 21,60 & 23,12 & 22,11 & 38,01 & 40,70 \\
\hline CREA & $15 \%$ & CLIA & 4,06 & 7,32 & 12,93 & 3,87 & 6,97 & 12,31 \\
\hline DB & $20 \%$ & CLIA & 12,96 & 7,87 & 16,72 & 12,67 & 7,69 & 16,34 \\
\hline GGT & $22,1 \%$ & RICOS & 6,65 & 13,80 & 12,50 & 11,08 & 23,01 & 20,85 \\
\hline GLU & $10 \%$ & CLIA & 9,08 & 16,45 & 26,42 & 9,91 & 17,95 & 28,83 \\
\hline HDL-C & $30 \%$ & CLIA & 15,40 & 32,61 & 26,80 & 16,92 & 35,85 & 29,46 \\
\hline Hierro & $20 \%$ & CLIA & 15,99 & 26,84 & 18,49 & 16,76 & 28,14 & 19,38 \\
\hline LDH & $20 \%$ & CLIA & 15,10 & 15,92 & 20,15 & 18,71 & 19,74 & 24,98 \\
\hline LDL-C & $20 \%$ & CAP & 19,69 & 29,09 & 13,02 & 19,22 & 28,40 & 12,72 \\
\hline $\mathrm{Mg}$ & $25 \%$ & CLIA & 11,09 & 12,88 & 12,84 & 11,92 & 13,84 & 13,79 \\
\hline$P$ & $10,7 \%$ & CAP & 4,94 & 7,48 & 10,28 & 5,17 & 7,87 & 10,77 \\
\hline BT & $20 \%$ & CLIA & 21,14 & 8,83 & 8,91 & 21,63 & 9,03 & 9,12 \\
\hline GT & $15 \%$ & NCEP & 5,54 & 12,58 & 13,91 & 5,99 & 13,61 & 15,04 \\
\hline PT & $10 \%$ & CLIA & 13,15 & 12,35 & 13,83 & 13,76 & 12,92 & 14,46 \\
\hline $\mathrm{AU}$ & $17 \%$ & CLIA & 7,80 & 17,98 & 20,36 & 7,80 & 17,99 & 20,38 \\
\hline \multicolumn{9}{|c|}{ Inmunoensayo } \\
\hline AFP & $20 \%$ & RCPA & 5,89 & 6,32 & 6,11 & 5,49 & 5,88 & 5,69 \\
\hline$\beta H C G$ & $30 \%$ & RiliBAK & 5,85 & 6,17 & 11,67 & 6,67 & 7,03 & 13,30 \\
\hline FER & $30 \%$ & CAP & 8,57 & 8,94 & 9,34 & 6,86 & 7,15 & 7,47 \\
\hline FSH & $20 \%$ & RCPA & 10,55 & 6,55 & 7,95 & 8,73 & 5,42 & 6,58 \\
\hline T3 libre & $17 \%$ & RICOS & 3,78 & 3,03 & 3,27 & 4,98 & 4,00 & 4,32 \\
\hline T4 libre & $16 \%$ & Spanish EQA Minimum & 3,42 & 8,21 & 4,05 & 4,78 & 11,47 & 5,66 \\
\hline $\mathrm{LH}$ & $20 \%$ & RCPA & 6,54 & 4,59 & 5,72 & 7,45 & 5,57 & 6,51 \\
\hline Prolactina & $20 \%$ & RCPA & 7,30 & 6,14 & 6,13 & 7,54 & 6,33 & 6,33 \\
\hline PSA total & $20 \%$ & Ricos Desirable & 12,07 & 8,66 & 7,27 & 11,98 & 8,59 & 7,22 \\
\hline TSH & $23,7 \%$ & CLIA & 8,70 & 6,72 & 9,92 & 6,81 & 5,26 & 7,76 \\
\hline VIT B12 & $30 \%$ & WSLH & 5,35 & 7,30 & 5,83 & 5,91 & 8,07 & 6,45 \\
\hline VIT D & $30 \%$ & Biological Variation Paper & 5,40 & 5,27 & 7,82 & 4,88 & 4,76 & 7,07 \\
\hline \multicolumn{9}{|l|}{ Electrolitos } \\
\hline $\mathrm{Cl}$ & $5 \%$ & CLIA & 5,39 & 4,94 & 5,98 & 5,53 & 5,06 & 6,13 \\
\hline $\mathrm{K}$ & $17,97 \%$ & CLIA & 9,45 & 14,96 & 20,84 & 9,46 & 14,97 & 20,86 \\
\hline $\mathrm{Na}$ & $3,57 \%$ & CLIA & 2,59 & 3,70 & 2,70 & 4,57 & 6,52 & 4,75 \\
\hline
\end{tabular}

$>6 \square \geq 3-6 \square<3 \quad$ ALB, albúmina; ALP, fosfatasa alcalina; ALT, alanina aminotransferasa; AMI, amilasa; AST, aspartato aminotransferasa; $\mathrm{Ca}$, calcio; $\mathrm{COL}$, colesterol; $\mathrm{CO}_{2}$, dióxido de carbono; $\mathrm{CPK}$, creatinina fosfoquinasa; $\mathrm{CREA}$, creatinina; $\mathrm{BD}$, bilirrubina directa; GGT, gamma glutamil transferasa; GLU, glucosa; HDL, lipoproteína de alta densidad; LDH, lactato deshidrogenasa; LDL, lipoproteína de baja densidad; Mg, magnesio; P, fósforo; PSA total, antígeno prostático específico total; BT, bilirrubina total; TG, triglicéridos; PT, proteína total; AU, ácido úrico; AFP, alfa fetoproteína; $\beta H C G$, gonadotropina coriónica humana beta; FER, ferritina; FSH, hormona estimulante del folículo; T3 libre, triyodotironina libre; T4 libre, tiroxina libre; HL, hormona luteinizante; TSH, hormona estimulante de la tiroides; VIT B12, vitamina B12; VIT D, vitamina D; Cl, cloruro; K, potasio; Na, sodio. CLIA 1992 fue la fuente de errores totales permitidos para distintas magnitudes. 


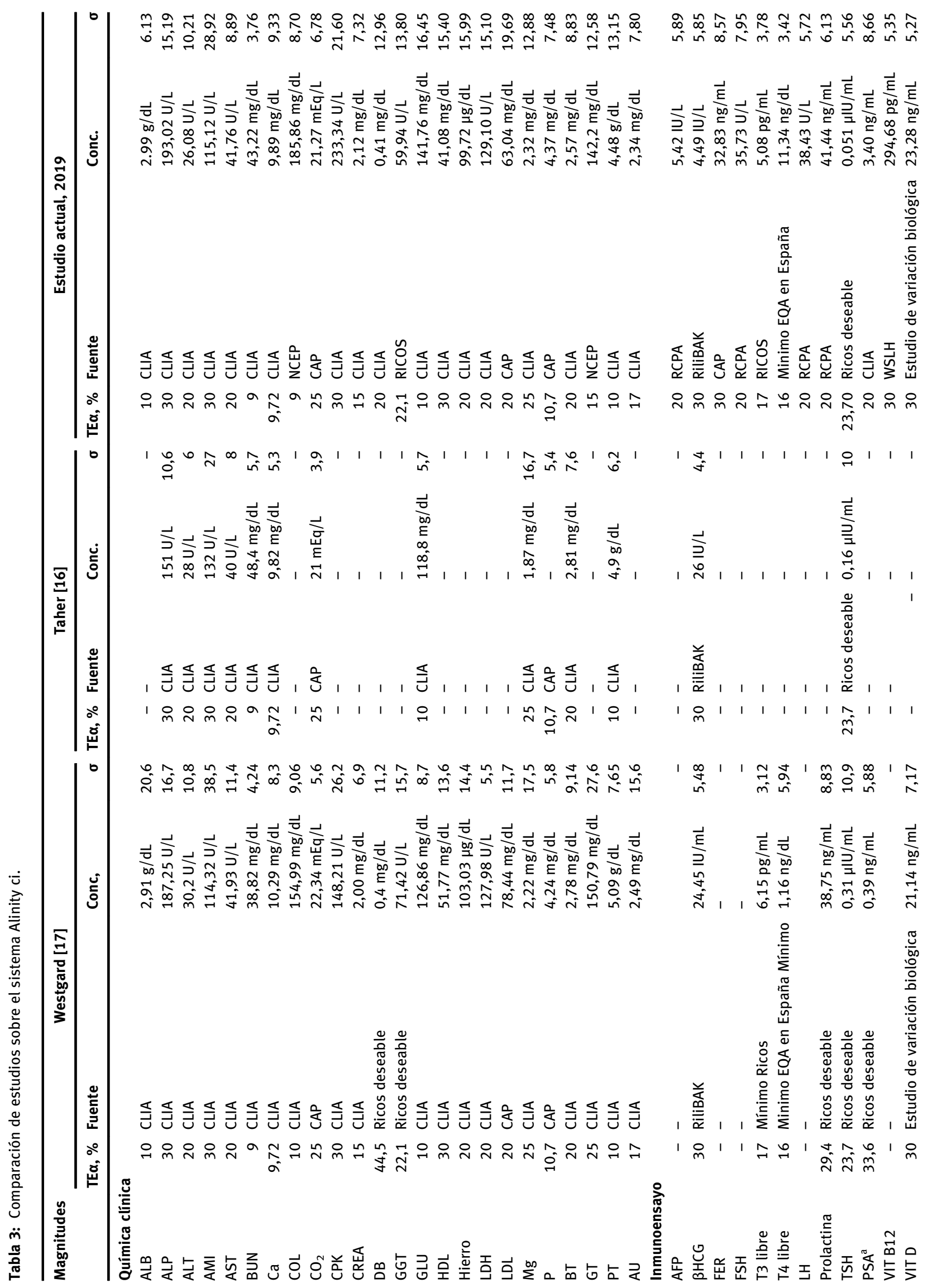




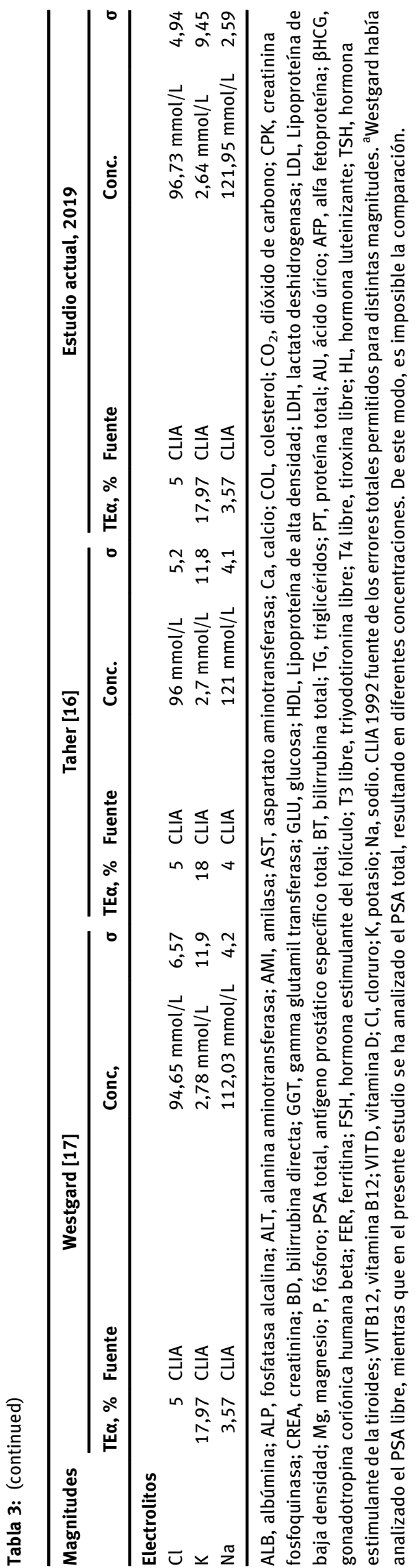

\section{Resultados}

En la Tabla 1 se pueden observar los niveles de concentración, precisión, coeficientes de variación y desviación de estas magnitudes. Como se puede ver, la precisión de todas las magnitudes es igual o inferior al 5,0\%, mientras que existe variabilidad en las desviaciones obtenidas con los distintos métodos. La precisión para las magnitudes químicas y los electrolitos supera a la de los inmunoensayos.

La Tabla 2 incluye un análisis de las métricas Sigma. Con respecto a los análisis clínicos, todos los parámetros excepto BUN, mostraron valores de Sigma $>6$ en uno o más niveles. Cabe señalar que se obtuvieron valores de Sigma $<6$ en uno o más niveles o métodos en $\mathrm{BUN}, \mathrm{CO}_{2}$, creatinina, fósforo y triglicéridos. Con respecto a los inmunoensayos, se obtuvieron valores de Sigma $>6$ en todos los niveles y con todos los métodos para la ferritina, prolactina y PSA, mientras que en otros parámetros se obtuvieron valores de Sigma > 6 en uno o más niveles en los diferentes métodos. No se obtuvo un valor de Sigma < 3 en ninguno de los parámetros de las analíticas clínicas. En relación a los electrolitos, el potasio obtuvo un valor Sigma $>6$ en todos los niveles y métodos, mientras que el sodio fue el único parámetro con un valor de Sigma <3 en dos niveles en el método de evaluación de rendimiento (Tabla 2).

Tal como se muestra en la Tabla 3, los valores de Sigma obtenidos en este estudio son comparables a los documentados en otros estudios sobre Alinity. En las Figuras 1 y S1 se detallan las Method Decision Charts (MEDx) realizadas con las librerías Matplotlib en Python3.6 en tres niveles de concentración con los tres métodos.

\section{Discusión}

La métrica Sigma es una herramienta sencilla y objetiva para el seguimiento y cuantificación de la calidad de las pruebas de laboratorio. Estos valores se pueden calcular fácilmente a partir de los valores de los controles de calidad ordinarios generados en los laboratorios, sin que ello suponga ningún coste adicional. Además, se puede utilizar para diseñar la política de control interno de calidad y reducir la frecuencia y número de controles diarios, lo que supone un ahorro en costes. Ante la actual crisis económica mundial, en la que los laboratorios tratan de alcanzar un equilibrio entre la aplicación de medidas de control de calidad y los costes que ello implica, ha surgido una nueva aplicación de la métrica Sigma basada en reglas de control de calidad.

Nuestros estudios iniciales de validación en Alinity ci (Abbott Diagnostics, Abbott Gmbh \& Co. KG, Wiesbaden, Germany) han arrojado resultados prometedores y 

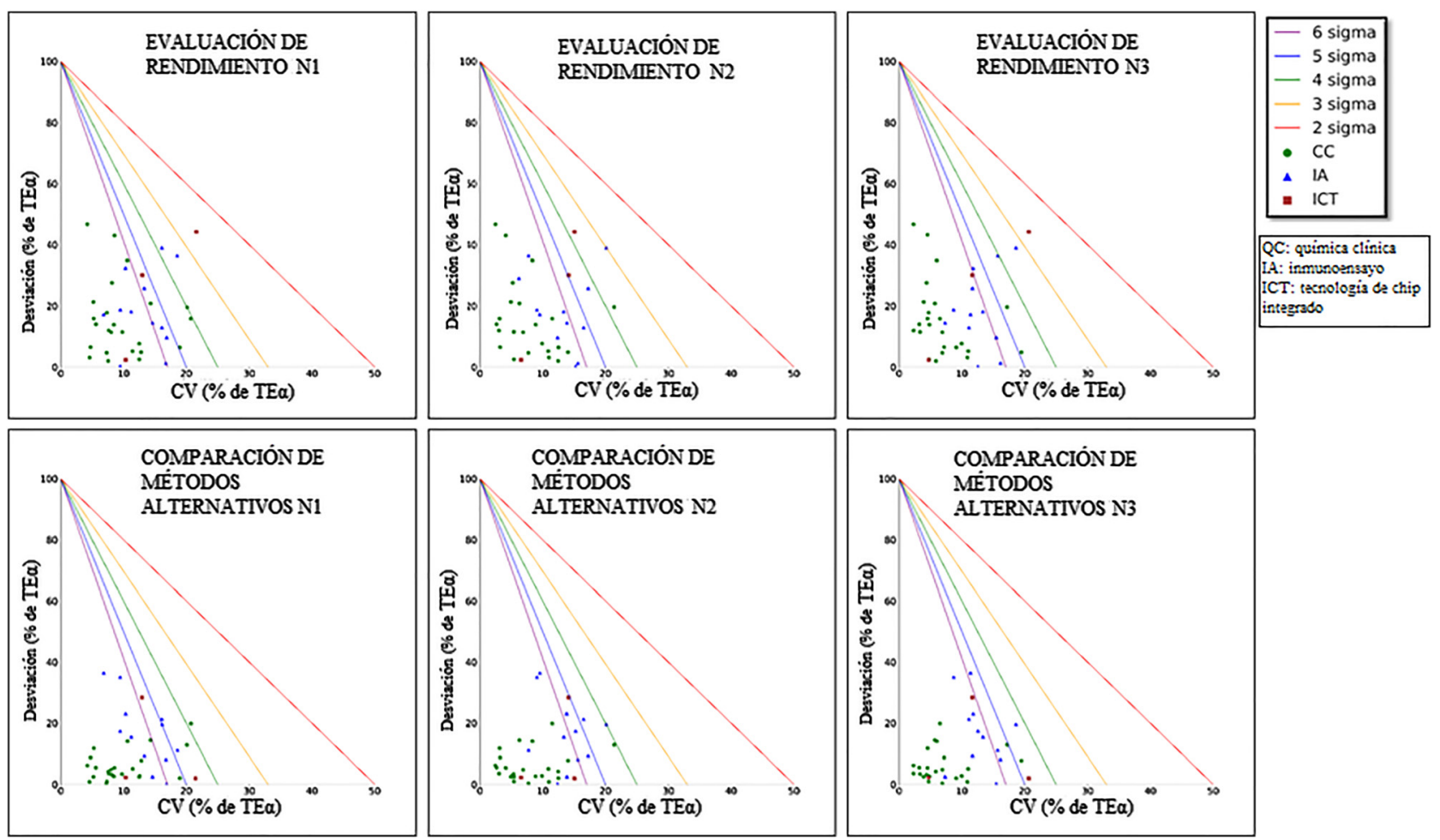

Figura 1: Gráficos de decisión de método normalizados en los que se muestran las métricas de Sigma empleando dos métodos diferentes.

muestran valores de Sigma elevados para la mayoría de las pruebas. Los tres métodos mostraron un buen rendimiento, aunque se obtuvieron resultados inferiores con el método de evaluación de rendimiento. Esto podría deberse a que las muestras se almacenaron a $-40{ }^{\circ} \mathrm{C}$ previamente a su análisis. Para compensar esta limitación, primero se confirmó la integridad de la muestra analizando las muestras en los sistemas existentes y comparando los resultados con los resultados resumidos del paciente. A continuación, se volvieron a analizar en un margen inferior a las dos horas en el sistema Alinity ci. Aunque para la obtención de valores de Sigma se realizaron una evaluación de rendimiento, una comparación de métodos alternativos y estudios de linealidad, recomendamos usar la evaluación de rendimiento como la herramienta más estandarizada para comparar de forma continuada el método de un laboratorio con los de otros laboratorios de cualquier parte del mundo. La comparación de métodos alternativos resulta útil durante la fase de evaluación inicial, aunque a veces resulta difícil emplearla en los inmunoensayos, aunque los estudios de linealidad son más útiles a la hora de medir rangos, así como en los estudios de verificación y calibración, que para calcular desviaciones.

La elección del error total permitido es el principal determinante a la hora de calcular valores de Sigma [19]. Cuando más amplio sea el límite, mayor será el valor de
Sigma, causando una falsa sensación de seguridad y derivando en una sobreestimación de la calidad de la prueba. El empleo de límites más exigentes reduce los niveles de Sigma, pero otorga una imagen más veraz de la calidad de la prueba. Hemos tratado de emplear TE $\alpha$ similares o inferiores a los utilizados en estudios comparativos sobre Alinity ci. Westgard [17] y Taher et al. [16] realizaron una secuencia en la que utilizaron los límites de error más bajos que se pueden obtener en las pruebas en un contexto real. Aplicamos los mismos límites, con el fin de comparar la escala Sigma obtenida con los análisis iniciales realizados según especificaciones factoriales, o sobre el campo por los investigadores de la compañía. En algunas pruebas como la bilirrubina directa ( $20 \%$ frente a $44.5 \%)$, triglicéridos (15\% frente a $25 \%)$ y prolactina $(20 \%$ frente a $29.4 \%$ ), elegimos unos límites de error permitido inferiores a los seleccionados en los estudios de referencia, obteniendo aún así el nivel seis Sigma. Los objetivos de error total permitido propuestos por CLIA en 2019 son un paso más para elevar el listón, y es nuestra intención emplearlos en futuros estudios.

Otro elemento del presente estudio a tener en cuenta es el número de magnitudes incluidas. Así, se incluyeron múltiples magnitudes que no fueron analizadas en los estudios previos de Westgard et al. [17] (AFP, ferritina, FSH, LH y vitamina B12) [16] (albúmina, colesterol, CPK, 
creatinina, bilirrubina directa, GGT, HDL, hierro, LDH, LDL, triglicéridos, ácido úrico, AFP, ferritina, FSH, FT3, FT4, LH, prolactina, PSA, vitaminas B12 y D).

Los analitos de química clínica suelen mostrar valores de Sigma más altos que los parámetros de inmunoensayo. Esto se debe a la mayor precisión de estas pruebas, en comparación con los inmunoensayos. La variabilidad de los niveles Sigma entre los diferentes métodos se debe a la variación en las desviaciones generadas, ya que la precisión empleada en los cálculos fue constante, ya que se obtuvo a partir de datos de controles internos de calidad.

El presente estudio presenta algunas limitaciones. En primer lugar, los resultados de las evaluaciones de rendimiento se compararon con los obtenidos en grupos de comparación de Architect debido al hecho de que no se disponía de los resultados de participación del CAP sobre Alinity en el momento de la realización de este estudio. En segundo lugar, este estudio se realizó en un periodo corto de tiempo con EP15 para la generación de datos de precisión y empleando un único lote de reactivos, calibradores y controles. La experiencia actual tras la implementación de estas pruebas ha cambiado completamente, disponiendo de análisis de grupos de comparación en tiempo real en encuestas del CAP, y con distintos lotes de reactivos, calibradores, controles, condiciones ambientales y operadores.

\section{Conclusiones}

El sistema Alinity ci generó una métrica Sigma aceptable para todos los analitos químicos clínicos y de inmunoensayo habitualmente utilizados en la práctica clínica. La métrica Sigma es una herramienta útil para evaluar la calidad de una magnitud biológica basándonos en su precisión y exactitud, que permitirá a los laboratorios modificar y espaciar la frecuencia de los controles internos de calidad. También permitirá a los laboratorios reducir costes sin comprometer la seguridad del paciente. En este estudio se aplicaron diferentes límites de error total aceptable. No obstante, con la introducción de los objetivos de error total permitido de CLIA de 2019, proponemos que estos sean adoptados por todos los laboratorios para calcular sus métricas Sigma.

Agradecimientos: Agradecemos a Sumbul Zehra su contribución en el análisis de datos y la elaboración de gráficas. Financiación de la investigación: No declarada. Contribución de los autores: Todos los autores aceptan la responsabilidad sobre la totalidad del contenido del manuscrito enviado, habiendo todos aprobado su presentación.

Conflicto de intereses: Los autores declaran no tener ningún conflicto de intereses.

Aprobación ética: Dado que este estudio no implica experimentación con seres humanos o animales, estaba exenta de aprobación por el Comité Ético correspondiente.

\section{Referencias}

1. Westgard S, Bayat H, Westgard JO. Analytical Sigma metrics: a review of Six Sigma implementation tools for medical laboratories. Biochem Med 2018;3:2-1.

2. Westgard J, Klee G. Quality management. In: Burtis C, Ashwood E, Bruns D, editors. Tietz textbook of clinical chemistry molecular Diagnostics, 4th ed. St Louis, MO: Elsevier Saunders; 2006: 498-9 pp.

3. Guo X, Zhang T, Gao X, Li PTY, Wu Q, Wu J, et al. Sigma metrics for assessing the analytical quality of clinical chemistry assays: a comparison of two approaches. Biochem Med 2018;28:204-14.

4. Li R, Wang T, Gong L, Peng P, Yang S, Zhao H, et al. Comparative analysis of calculating Sigma metrics by a trueness verification proficiency testing-based approach and an internal quality control data inter-laboratory comparison-based approach. J Clin Lab Anal 2019;33:e22989.

5. RCPA allowable limits of performance for biochemistry; 2014 . Available from: http://www.rcpaqap.com.au/docs/2014/ chempath/ALP.pdf.

6. Pathologists CoA. Proficiency testing; 2019. Available from: https:// www.cap.org/laboratory-improvement/proficiency-testing.

7. Association GM. Revision of the "guideline of the German Medical Association on quality assurance in medical laboratory examinations-RILIBAEK". Lab Med 2015;39:26-69.

8. Data Innovations. Allowable total error table. Available from: www.datainnovations.com/allowable-total-error-table.

9. Lepor NE, Vogel RE. Summary of the third report of the national cholesterol education program adult treatment panel III. Rev Cardiovasc Med 2019;2:160-5.

10. Viljoen A, Singh DK, Farrington K, Twomey PJ. Analytical quality goals for 25-vitamin D based on biological variation. J Clin Lab Anal 2011;25:130-3.

11. Coskun A, Inal T, Unsal I, Serteser M. Six Sigma as a quality management tool: evaluation of performance in laboratory medicine. Qual Manag Six Sigma 2010;9928:247-61.

12. Coskun A. Six Sigma and laboratory consultation. Clin Chem Lab Med 2007;45:121-3.

13. Rifai N, Horvath A, Wittwer C. Tietz textbook of clinical chemistry and molecular diagnostics. Missouri: Elsevier Health Sciences; 2018.

14. Westgard JO, Westgard SA. Quality control review: implementing a scientifically based quality control system. Ann Clin Biochem 2016;53:32-50.

15. Westgard JO, Bayat $\mathrm{H}$, Westgard SA. Planning risk-based SQC schedules for bracketed operation of continuous production analyzers. Ann Clin Biochem 2018;64:289-96. 
16. Taher J, Cosme J, Renley BA, Daghfal DJ, Yip PM. A novel Sigma metric encompasses global multi-site performance of 18 assays on the Abbott Alinity system. Clin Biochem 2019;63:106-12.

17. Westgard S, Petrides V, Schneider S, Berman M, Herzogenrath J, Orzechowski A. Assessing precision, bias and Sigma-metrics of 53 measurands of the Alinity ci system. Clin Biochem 2017;50:1216-21.

18. Institute CLS. User verification of performance for precision and trueness; approved guideline. CLSI Document EP15-A2, 2005.

19. Hens K, Berth M, Armbruster D, Westgard S. Sigma metrics used to assess analytical quality of clinical chemistry assays: importance of the allowable total error (TEa) target. Clin Chem Lab Med 2014;52:973-80.

Material Suplementario: La versión en línea de este artículo ofrece material suplementario (https://doi.org/10.1515/almed-2021-0025).

Nota del artículo: El artículo original puede encontrarse aquí: https:// doi.org/10.1515/almed-2021-0001. 